\title{
On Type 2 Degenerate Poly-Frobenius-Genocchi Polynomials and Numbers
}

\author{
Ugur Duran $^{a}$, Mehmet Acikgoz ${ }^{b}$ and Serkan Araci ${ }^{c, *}$ \\ ${ }^{a}$ Department of Basic Sciences of Engineering, \\ Faculty of Engineering and Natural Sciences, \\ Iskenderun Tecnical University, TR-31200 Hatay, Turkey \\ E-Mail: mtdrnugur@gmail.com \\ ${ }^{b}$ Department of Mathematics, Faculty of Arts and Science, \\ University of Gaziantep, TR-27310 Gaziantep, Turkey \\ E-Mail: acikgoz@gantep.edu.tr \\ ${ }^{c}$ Department of Economics, Faculty of Economics, \\ Administrative and Social Science, \\ Hasan Kalyoncu University, TR-27410 Gaziantep, Turkey \\ E-Mail: mtsrkn@hotmail.com \\ ${ }^{*}$ Corresponding Author
}

\begin{abstract}
In this paper, we consider a class of new generating function for the Frobenius-Genocchi polynomials, called the type 2 degenerate poly-Frobenius-Genocchi polynomials, by means of the polyexponential function. Then, we investigate diverse explicit expressions and some identities for those polynomials.
\end{abstract}

2010 Mathematics Subject Classification- 11B83, 11S80, 05A19.

Keywords and Phrases- Polyexponential function; Degenerate exponential function; Polyexponential function; Frobenius-Genocchi polynomials; Poly-Frobenius-Genocchi polynomials.

\section{INTRODUCTION}

Special polynomials have their origin in the solution of the differential equations (or partial differential equations) under some conditions. Special polynomials can be defined in a various ways such as by generating functions, by recurrence relations, by $p$-adic integrals in the sense of fermionic and bosonic, by degenerate versions, etc.

Kim-Kim have introduced polyexponential function in [18] and its degenerate version in [20],[21]. By making use of aforementioned function, they have introduced a new class of some special polynomials. This idea provides a powerfool tool in order to define special numbers and polynomials by making use of polyexponential function. One may see that the notion of polyexponential function form a special class of polynomials because of their great applicability, cf. [12, 18-22, 26, 27, 29,31]. The importance of these polynomials would be to find applications in analytic number theory, applications in classical analysis and statistics, cf. [1-34].

Throughout of the paper we make use of the following notations: $\mathbb{N}:=\{1,2,3, \cdots\}$ and $\mathbb{N}_{0}=\mathbb{N} \cup\{0\}$. Here, as usual, $\mathbb{Z}$ denotes the set of integers, $\mathbb{R}$ denotes the set of real numbers and $\mathbb{C}$ denotes the set of complex numbers.

The classical Bernoulli $B_{n}(x)$, Euler $E_{n}(x)$ and Genocchi $G_{n}(x)$ polynomials and the degenerate Bernoulli $B_{n, \lambda}(x)$, Euler $E_{n, \lambda}(x)$ and Genocchi $G_{n, \lambda}(x)$ polynomials are given as follows $(c f . \quad[5,8,10,11,14,16$, 18-20, 22, 23, 26-32]):

$$
\begin{aligned}
& \sum_{n=0}^{\infty} B_{n}(x) \frac{t^{n}}{n !}=\frac{t}{e^{t}-1} \text { and } \sum_{n=0}^{\infty} B_{n, \lambda}(x) \frac{t^{n}}{n !}=\frac{t}{e_{\lambda}(t)-1} e_{\lambda}^{x}(t) \\
& \sum_{n=0}^{\infty} E_{n}(x) \frac{t^{n}}{n !}=\frac{2}{e^{t}+1} \text { and } \sum_{n=0}^{\infty} E_{n, \lambda}(x) \frac{t^{n}}{n !}=\frac{2}{e_{\lambda}(t)+1} e_{\lambda}^{x}(t)
\end{aligned}
$$




$$
\sum_{n=0}^{\infty} G_{n}(x) \frac{t^{n}}{n !}=\frac{2 t}{e^{t}+1} \text { and } \sum_{n=0}^{\infty} G_{n, \lambda}(x) \frac{t^{n}}{n !}=\frac{2 t}{e_{\lambda}(t)+1} e_{\lambda}^{x}(t) .
$$

One may look at the references $[1,4-13,15,17-19,21,22,25-31]$ to see the various applications of Bernoulli, Euler and Genocchi polynomials.

Frobenius studied the polynomials $F_{n}(x \mid u)$ given by $(c f .[2,3])$

$$
\frac{1-u}{e^{t}-u} e^{x t}=\sum_{n=0}^{\infty} F_{n}(x \mid u) \frac{t^{n}}{n !} \quad(u \in \mathbb{C} \backslash\{1\}) .
$$

Upon setting $u=-1$, it becomes

$$
F_{n}(x \mid-1)=E_{n}(x)
$$

Owing to relationship with the Euler polynomials as well as their important properties, and in the honor of Frobenius, the aforementioned polynomials denoted by $F_{n}(x \mid u)$ are called the Frobenius-Euler polynomials, cf. $[2,3]$. by

Parallel to (1.4), Yaşar and Özarslan [34] introduced the Frobenius-Genocchi polynomials $G_{n}^{F}(x ; u)$ given

$$
\frac{(1-u) t}{e^{t}-u} e^{x t}=\sum_{n=0}^{\infty} G_{n}^{F}(x ; u) \frac{t^{n}}{n !},
$$

since

$$
G_{n}^{F}(x ;-1)=G_{n}(x) .
$$

The case $x=0$ in $(1.5), G_{n}^{F}(0 ; u):=G_{n}^{F}(u)$ stands for the Frobenius-Genocchi numbers. Several recurrence relations and differential equations are also investigated in [34].

Khan and Srivastava [17] introduced a new class of the generalized Apostol type Frobenius-Genocchi polynomials and investigated some properties and relations including implicit summation formulae and various symmetric identities. Moreover a relation in between Array-type polynomials, Apostol-Bernoulli polynomials and generalized Apostol-type Frobenius-Genocchi polynomials is also given in [17]. Wani et al. [33] considered Gould-Hopper based Frobenius-Genocchi polynomials and then, summation formulae and operational rule for these polynomials.

The Bernoulli polynomials of the second kind are defined by means of the following generating function

$$
\sum_{n=0}^{\infty} b_{n}(x) \frac{t^{n}}{n !}=\frac{t}{\log (1+t)}(1+t)^{x} .
$$

When $x=0, b_{n}(0):=b_{n}$ are called the Bernoulli numbers of the second kind, $c f$. [20].

It is well-known from (1.6) that

$$
\left(\frac{t}{\log (1+t)}\right)^{r}(1+t)^{x-1}=\sum_{n=0}^{\infty} B_{n}^{(n-r+1)}(x) \frac{t^{n}}{n !},
$$

where $B_{n}^{(r)}(x)$ are the Bernoulli polynomials of order $r$, see [20].

For $\lambda \in \mathbb{C}$, the $\lambda$-falling factorial $(x)_{n, \lambda}$ is defined by (see $[10,11,20-22,24-27,29-31]$ )

$$
(x)_{n, \lambda}= \begin{cases}x(x-\lambda)(x-2 \lambda) \cdots(x-(n-1) \lambda), & n=1,2, \ldots \\ 1 & n=0 .\end{cases}
$$

In the case $\lambda=1$, the $\lambda$-falling factorial reduces to the familiar falling factorial as follows

$$
(x)_{n, 1}:=(x)_{n}=x(x-1) \cdots(x-n+1) \text { and }(x)_{0}=1 .
$$

The $\Delta_{\lambda}$ difference operator is defined by (see $[10,11]$ )

$$
\Delta_{\lambda} f(x)=\frac{1}{\lambda}(f(x+\lambda)-f(x)), \quad \lambda \neq 0 .
$$


The degenerate exponential function $e_{\lambda}^{x}(t)$ is defined as follows

$$
e_{\lambda}^{x}(t)=(1+\lambda t)^{\frac{x}{\lambda}} \text { and } e_{\lambda}^{1}(t)=e_{\lambda}(t) .
$$

It is readily seen that $\lim _{\lambda \rightarrow 0} e_{\lambda}^{x}(t)=e^{x t}(c f .[10,11,20-22,24-27,29-31])$. From (1.8) and (1.10), we obtain the following relation

$$
e_{\lambda}^{x}(t)=\sum_{n=0}^{\infty}(x)_{n, \lambda} \frac{t^{n}}{n !}
$$

which satisfies the following difference rule

$$
\Delta_{\lambda} e_{\lambda}^{x}(t)=t e_{\lambda}^{x}(t)
$$

The Stirling numbers of the first kind $S_{1}(n, k)$ and the Stirling numbers of the second kind $S_{2}(n, k)$ are defined $(c f .[2,4,5,12])$ by means of the following generating functions:

$$
\frac{(\log (1+t))^{k}}{k !}=\sum_{n=0}^{\infty} S_{1}(n, k) \frac{t^{n}}{n !} \text { and } \frac{\left(e^{t}-1\right)^{k}}{k !}=\sum_{n=0}^{\infty} S_{2}(n, k) \frac{t^{n}}{n !} .
$$

From (1.13), we get the following relations for $n \geq 0$ :

$$
(x)_{n}=\sum_{k=0}^{n} S_{1}(n, k) x^{k} \text { and } x^{n}=\sum_{k=0}^{n} S_{1}(n, k)(x)_{k} .
$$

Very recently, Kim-Kim [22] performed to generalize the degenerate Bernoulli polynomials by using polyexponential function

$$
\operatorname{Ei}_{k}(t)=\sum_{n=1}^{\infty} \frac{t^{n}}{(n-1) ! n^{k}}
$$

as inverse to the polylogarithm function

$$
L i_{k}(t)=\sum_{n=1}^{\infty} \frac{t^{n}}{n^{k}} \quad(|t|<1 ; k \in \mathbb{Z})
$$

given by

$$
\frac{\operatorname{Ei}_{k}(\log (1+t))}{e_{\lambda}(t)-1} e_{\lambda}^{x}(t)=\sum_{n=0}^{\infty} \beta_{n, \lambda}^{(k)}(x) \frac{t^{n}}{n !} .
$$

Upon setting $x=0$ in (1.17), $\beta_{n, \lambda}^{(k)}(0):=\beta_{n, \lambda}^{(k)}$ are called the degenerate poly-Bernoulli numbers. Kim et al. [22] studied the degenerate poly-Bernoulli polynomials and also gave some explicit expressions and several formulas for those polynomials.

For $k \in \mathbb{Z}$, the type 2 degenerate poly-Euler polynomials $\mathfrak{E}_{n, \lambda}^{(k)}(x)$ are defined, $c f$. [29], as follows:

$$
\frac{\operatorname{Ei}_{k}(\log (1+2 t))}{t\left(e_{\lambda}(t)+1\right)} e_{\lambda}^{x}(t)=\sum_{n=0}^{\infty} \mathfrak{E}_{n, \lambda}^{(k)}(x) \frac{t^{n}}{n !}
$$

When $x=0, \mathfrak{E}_{n, \lambda}^{(k)}(0):=\mathfrak{E}_{n, \lambda}^{(k)}$ are called the type 2 degenerate poly-Euler numbers. Lee et al. [29] studied the type 2 degenerate poly-Euler polynomials and provided multifarious explicit formulas and identities.

Since $\operatorname{Ei}_{1}(t)=e^{t}-1$, it is worthy to note that

$$
\beta_{n, \lambda}^{(1)}(x):=B_{n, \lambda}(x) \text { and } \mathfrak{E}_{n, \lambda}^{(1)}(x):=E_{n, \lambda}(x) .
$$




\section{The type 2 Degenerate Poly-Frobenius-Genocchi Polynomials}

Now, we consider the following Definition 1 by means of the polyexponential function.

Definition 1. Let $k \in \mathbb{Z}$. The type 2 degenerate poly-Frobenius-Genocchi polynomials are defined via the following exponential generating function (in a suitable neigbourhood of $t=0$ ) including the polyexponential function as given below:

$$
\frac{\operatorname{Ei}_{k}(\log (1+(1-u) t))}{e_{\lambda}(t)-u} e_{\lambda}^{x}(t)=\sum_{n=0}^{\infty} G_{n, \lambda}^{(F, k)}(x ; u) \frac{t^{n}}{n !} .
$$

At the value $x=0$ in (2.1), $G_{n, \lambda}^{(F, k)}(0 ; u):=G_{n, \lambda}^{(F, k)}(u)$ will be called type 2 degenerate poly-Frobenius-Genocchi numbers.

Remark 1. Taking $k=1$ in (2.1) yields $G_{n, \lambda}^{(F, 1)}(x ; u):=G_{n, \lambda}^{F}(x ; u)$ are the degenerate Frobenius-Genocchi polynomials $G_{n, \lambda}^{F}(x ; u)$ (cf. [15]) as follows

$$
\frac{(1-u) t}{e_{\lambda}(t)-u} e_{\lambda}^{x}(t)=\sum_{n=0}^{\infty} G_{n, \lambda}^{F}(x ; u) \frac{t^{n}}{n !} .
$$

Remark 2. Upon setting $\lambda \rightarrow 0$ in (2.1) gives $\lim _{\lambda \rightarrow 0} G_{n, \lambda}^{(F, k)}(x ; u):=G_{n}^{(F, k)}(x ; u)$ are type 2 poly-FrobeniusGenocchi polynomials $G_{n}^{(F, k)}(x ; u)$ (cf. [12]) as follows

$$
\frac{\operatorname{Ei}_{k}(\log (1+(1-u) t))}{e^{t}-u} e^{x t}=\sum_{n=0}^{\infty} G_{n}^{(F, k)}(x ; u) \frac{t^{n}}{n !} .
$$

Remark 3. Taking $k=1$ and $\lambda \rightarrow 0$ in (2.1) yields $G_{n, \lambda}^{(F, 1)}(x ;-1):=G_{n, \lambda}(x)$ are the Frobenius-Genocchi polynomials in (1.5).

A difference operator rule of type 2 degenerate poly-Frobenius-Genocchi polynomials is given as follows

$$
\Delta_{\lambda} G_{n, \lambda}^{(F, k)}(x ; u)=G_{n-1, \lambda}^{(F, k)}(x ; u) .
$$

Now, we give the following theorem.

Theorem 1. The following relation

$$
G_{n, \lambda}^{(F, k)}(x ; u)=\sum_{l=0}^{n}\left(\begin{array}{c}
n \\
l
\end{array}\right) G_{n-l, \lambda}^{(F, k)}(u)(x)_{l, \lambda}
$$

is valid for $k \in \mathbb{Z}$ and $n \geq 0$.

Proof. By Definition 1, we consider that

$$
\begin{aligned}
\sum_{n=0}^{\infty} G_{n, \lambda}^{(F, k)}(x ; u) \frac{t^{n}}{n !} & =\frac{\operatorname{Ei}_{k}(\log (1+(1-u) t))}{e_{\lambda}(t)-u} e_{\lambda}^{x}(t) \\
& =\left(\sum_{n=0}^{\infty} G_{n, \lambda}^{(F, k)}(u) \frac{t^{n}}{n !}\right)\left(\sum_{n=0}^{\infty}(x)_{n, \lambda} \frac{t^{n}}{n !}\right) \\
& =\sum_{n=0}^{\infty}\left(\sum_{l=0}^{n}\left(\begin{array}{c}
n \\
l
\end{array}\right) G_{n-l, \lambda}^{(F, k)}(u)(x)_{l, \lambda}\right) \frac{t^{n}}{n !},
\end{aligned}
$$

which implies the asserted result in (2.2).

Now, we give the following theorem. 
Theorem 2. The following relation

$$
\frac{d}{d x} G_{n, \lambda}^{(F, k)}(x ; u)=n ! \sum_{u=1}^{\infty} G_{n-u, \lambda}^{(F, k)}(x ; u) \frac{(-1)^{u+1}}{(n-u) ! u} \lambda^{u-1}
$$

is valid for $k \in \mathbb{Z}$ and $n \geq 0$.

Proof. By Definition 1, we consider that

$$
\begin{aligned}
\sum_{n=0}^{\infty} \frac{d}{d x} G_{n, \lambda}^{(F, k)}(x ; u) \frac{t^{n}}{n !} & =\frac{\operatorname{Ei}_{k}(\log (1+(1-u) t))}{e_{\lambda}(t)-u} \frac{d}{d x} e_{\lambda}^{x}(t) \\
& =\sum_{n=0}^{\infty} G_{n, \lambda}^{(F, k)}(x ; u) \frac{t^{n}}{n !} \frac{1}{\lambda} \ln (1+\lambda t) \\
& =\left(\sum_{n=0}^{\infty} G_{n, \lambda}^{(F, k)}(x ; u) \frac{t^{n}}{n !}\right) \sum_{u=1}^{\infty} \frac{(-1)^{u+1}}{u} \lambda^{u-1} t^{u} \\
& =\sum_{n=0}^{\infty} \sum_{u=1}^{\infty} G_{n, \lambda}^{(F, k)}(x ; u) \frac{(-1)^{u+1}}{u} \lambda^{u-1} \frac{t^{n+u}}{n !}
\end{aligned}
$$

which implies the asserted result in (2.2).

A relation between the type 2 degenerate poly-Frobenius-Genocchi polynomials and the degenerate Frobenius-Genocchi polynomials is stated in the following theorem.

Theorem 3. For $k \in \mathbb{Z}$ and $n \geq 0$, we have

$$
G_{n, \lambda}^{(F, k)}(x ; u)=\sum_{m=0}^{n} \sum_{l=1}^{m+1}\left(\begin{array}{c}
n \\
m
\end{array}\right) \frac{S_{1}(m+1, l)(1-u)^{m}}{l^{k-1}(m+1)} G_{n-m, \lambda}^{F}(x ; u) .
$$

Proof. From (1.15), we observe that

$$
\begin{aligned}
\operatorname{Ei}_{k}(\log (1+(1-u) t)) & =\sum_{l=1}^{\infty} \frac{(\log (1+(1-u) t))^{l}}{(l-1) ! l^{k}} \\
& =\sum_{l=1}^{\infty} \frac{1}{l^{k-1}} \sum_{m=l}^{\infty} S_{1}(m, l)(1-u)^{m} \frac{t^{m}}{m !} \\
& =\sum_{m=0}^{\infty} \sum_{l=1}^{m+1} \frac{S_{1}(m+1, l)(1-u)^{m+1}}{l^{k-1}(m+1)} \frac{t^{m+1}}{m !}
\end{aligned}
$$

Then, by (2.1), we get

$$
\begin{gathered}
\frac{t(1-u)}{e_{\lambda}(t)-u} e_{\lambda}^{x}(t) \frac{1}{t(1-u)} \operatorname{Ei}_{k}(\log (1+(1-u) t))=\sum_{n=0}^{\infty} G_{n, \lambda}^{(F)}(x ; u) \frac{t^{n}}{n !} \\
\times \sum_{m=0}^{\infty} \sum_{l=1}^{m+1} \frac{S_{1}(m+1, l)(1-u)^{m}}{l^{k-1}(m+1)} \frac{t^{m}}{m !} \\
=\sum_{n=0}^{\infty}\left(\sum_{m=0}^{n} \sum_{l=1}^{m+1}\left(\begin{array}{c}
n \\
m
\end{array}\right) \frac{S_{1}(m+1, l)(1-u)^{m}}{l^{k-1}(m+1)} G_{n-m, \lambda}^{F}(x ; u)\right) \frac{t^{n}}{n !} .
\end{gathered}
$$

which means the asserted result in (2.4).

The immediate results of the Theorem 3 are stated below. 
Corollary 1. For $k \in \mathbb{Z}$ and $n \geq 0$, we have

$$
G_{n, \lambda}^{(F, k)}(u)=\sum_{m=0}^{n} \sum_{l=1}^{m+1}\left(\begin{array}{c}
n \\
m
\end{array}\right) \frac{S_{1}(m+1, l)(1-u)^{m}}{l^{k-1}(m+1)} G_{n-m, \lambda}^{F}(u) .
$$

Corollary 2. Taking $k=1$ in Theorem 3 gives

$$
G_{n, \lambda}^{(F, 1)}(x ; u)=\sum_{m=0}^{n} \sum_{l=1}^{m+1}\left(\begin{array}{c}
n \\
m
\end{array}\right) \frac{S_{1}(m+1, l)(1-u)^{m}}{(m+1)} G_{n-m, \lambda}^{F}(x ; u) .
$$

Corollary 3. Taking $k=1$ and $u=-1$ in Theorem 3 reduces

$$
G_{n, \lambda}(x)=\sum_{m=0}^{n} \sum_{l=1}^{m+1}\left(\begin{array}{c}
n \\
m
\end{array}\right) \frac{S_{1}(m+1, l)(1-u)^{m}}{(m+1)} G_{n-m, \lambda}(x) .
$$

Here, we give the following lemma.

Lemma 1. For $k \in \mathbb{Z}$ and $n \geq 0$, we have

$$
\frac{d}{d x} \operatorname{Ei}_{k}(\log (1+(1-u) x))=\frac{1-u}{(1+(1-u) x) \log (1+(1-u) x)} \operatorname{Ei}_{k-1}(\log (1+(1-u) x)) .
$$

Proof. From (1.15), we observe that

$$
\begin{aligned}
\frac{d}{d x} \operatorname{Ei}_{k}(\log (1+(1-u) x)) & =\frac{d}{d x} \sum_{l=1}^{\infty} \frac{(\log (1+(1-u) x))^{l}}{(l-1) ! l^{k}} \\
& =\frac{1-u}{(1+(1-u) x) \log (1+(1-u) x)} \sum_{l=1}^{\infty} \frac{(\log (1+(1-u) x))^{l}}{(l-1) ! l^{k-1}} \\
& =\frac{1-u}{(1+(1-u) x) \log (1+(1-u) x)} \operatorname{Ei}_{k-1}(\log (1+(1-u) x)),
\end{aligned}
$$

which is the claimed result in (2.7).

Theorem 4. Let $k \geq 2$. We have

$$
\begin{aligned}
G_{n, \lambda}^{(F, k)}(u)= & \sum_{m=0}^{n}\left(\begin{array}{c}
n \\
m
\end{array}\right) \sum_{m_{1}+m_{2}+\cdots+m_{k-1}=m}^{\infty}\left(\begin{array}{c}
m \\
m_{1}, m_{2}, \cdots, m_{k-1}
\end{array}\right)(1-u)^{m_{1}+m_{2}+\cdots m_{k-1}} \\
& \times G_{n-m, \lambda}^{F}(u) \frac{B_{m_{1}}^{\left(m_{1}\right)}(0)}{m_{1}+1} \frac{B_{m_{2}}^{\left(m_{2}\right)}(0)}{m_{1}+m_{2}+1} \cdots \frac{B_{m_{k-1}}^{\left(m_{k-1}\right)}(0)}{m_{1}+m_{2}+\cdots+m_{k-1}+1}
\end{aligned}
$$

Proof. By (2.7), we consider

$$
\begin{gathered}
\operatorname{Ei}_{k}(\log (1+(1-u) x))=\int_{0}^{x} \frac{1-u}{(1+(1-u) x) \log (1+(1-u) x)} \operatorname{Ei}_{k-1}(\log (1+(1-u) x)) d t \\
\quad=\int_{0}^{x} \frac{1-u}{(1+(1-u) x) \log (1+(1-u) x)} \\
\times \underbrace{\int_{0}^{t} \frac{1-u}{(1+(1-u) x) \log (1+(1-u) x)} \cdots \int_{0}^{t} \frac{(1-u)^{2} t}{(1+(1-u) x) \log (1+(1-u) x)}}_{(k-2) \text { times }} d t d t \cdots d t .
\end{gathered}
$$


Then, we obtain

$$
\begin{aligned}
& \sum_{n=0}^{\infty} G_{n, \lambda}^{(F, k)}(u) \frac{t^{n}}{n !}=\frac{\operatorname{Ei}_{k}(\log (1+(1-u) t))}{e_{\lambda}(t)-u} \\
& =\frac{1}{e_{\lambda}(t)-u} \int_{0}^{x} \frac{1-u}{(1+(1-u) x) \log (1+(1-u) x)} \\
& \times \underbrace{\int_{0}^{t} \frac{1-u}{(1+(1-u) x) \log (1+(1-u) x)} \cdots \int_{0}^{t} \frac{(1-u)^{2} t}{(1+(1-u) x) \log (1+(1-u) x)}}_{(k-2) \text { times }} d t d t \cdots d t \\
& =\frac{(1-u) x}{e_{\lambda}(t)-u} \sum_{m=0}^{\infty} \sum_{m_{1}+m_{2}+\cdots+m_{k-1}=m}^{\infty}\left(\begin{array}{c}
m \\
m_{1}, m_{2}, \cdots, m_{k-1}
\end{array}\right)(1-u)^{m_{1}+m_{2}+\cdots m_{k-1}} \\
& \times \frac{B_{m_{1}}^{\left(m_{1}\right)}(0)}{m_{1}+1} \frac{B_{m_{2}}^{\left(m_{2}\right)}(0)}{m_{1}+m_{2}+1} \cdots \frac{B_{m_{k-1}}^{\left(m_{k-1}\right)}(0)}{m_{1}+m_{2}+\cdots+m_{k-1}+1} \frac{x^{m}}{m !} \\
& =\sum_{n=0}^{\infty} \sum_{m=0}^{n}\left(\begin{array}{l}
n \\
m
\end{array}\right) \sum_{m_{1}+m_{2}+\cdots+m_{k-1}=m}^{\infty}\left(\begin{array}{c}
m \\
m_{1}, m_{2}, \cdots, m_{k-1}
\end{array}\right)(1-u)^{m_{1}+m_{2}+\cdots m_{k-1}} \\
& \times G_{n-m, \lambda}^{F}(u) \frac{B_{m_{1}}^{\left(m_{1}\right)}(0)}{m_{1}+1} \frac{B_{m_{2}}^{\left(m_{2}\right)}(0)}{m_{1}+m_{2}+1} \cdots \frac{B_{m_{k-1}}^{\left(m_{k-1}\right)}(0)}{m_{1}+m_{2}+\cdots+m_{k-1}+1} \frac{x^{n}}{n !}
\end{aligned}
$$

This finalizes the proof of the theorem.

Now, we give the following theorem.

Theorem 5. For $n \in \mathbb{N}_{0}$, we have

$$
\sum_{m=0}^{n} \frac{S_{2}(n, m)}{(1-u)^{m}} G_{m, \lambda}^{(F, k)}(u)=\sum_{m=0}^{n} \sum_{k=0}^{m} \sum_{j=0}^{k}\left(\begin{array}{c}
n \\
m
\end{array}\right)\left(\begin{array}{c}
m \\
k
\end{array}\right) \frac{G_{j, \lambda}^{(F)}(u) S_{2}(k, j)}{(1-u)^{j}} \frac{B_{m-k}}{(n-m+1)^{k}}
$$

Proof. Replacing $t$ by $\frac{e^{t}-1}{1-u}$ in (2.1), we attain

$$
\begin{aligned}
\frac{\operatorname{Ei}_{k}(t)}{e_{\lambda}\left(\frac{e^{t}-1}{1-u}\right)-u} & =\sum_{m=0}^{\infty}(1-u)^{-m} G_{m, \lambda}^{(F, k)}(u) \frac{\left(e^{t}-1\right)^{m}}{m !} \\
& =\sum_{m=0}^{\infty}(1-u)^{-m} G_{m, \lambda}^{(F, k)}(u) \sum_{n=0}^{\infty} S_{2}(n, m) \frac{t^{n}}{n !} \\
& =\sum_{n=0}^{\infty} \sum_{m=0}^{n} \frac{S_{2}(n, m)}{(1-u)^{m}} G_{m, \lambda}^{(F, k)}(u) \frac{t^{n}}{n !}
\end{aligned}
$$


Also, we investigate

$$
\begin{gathered}
\frac{(1-u)\left(\frac{e^{t}-1}{1-u}\right)}{e_{\lambda}\left(\frac{e^{t}-1}{1-u}\right)-u} \frac{1}{e^{t}-1} \sum_{l=1}^{\infty} \frac{t^{l}}{(l-1) ! l^{k}}=\frac{(1-u)\left(\frac{e^{t}-1}{1-u}\right)}{e_{\lambda}\left(\frac{e^{t}-1}{1-u}\right)-u} \frac{t}{e^{t}-1} \sum_{l=0}^{\infty} \frac{t^{l}}{l !(l+1)^{k}} \\
=\sum_{j=0}^{\infty}(1-u)^{-j} G_{j, \lambda}^{(F)}(u) \frac{\left(e^{t}-1\right)^{j}}{j !} \sum_{i=0}^{\infty} B_{i} \frac{t^{i}}{i !} \sum_{l=0}^{\infty} \frac{t^{l}}{l !(l+1)^{k}} \\
=\sum_{k=0}^{\infty} \sum_{j=0}^{k}(1-u)^{-j} G_{j, \lambda}^{(F)}(u) S_{2}(k, j) \frac{t^{k}}{k !} \sum_{i=0}^{\infty} B_{i} \frac{t^{i}}{i !} \sum_{l=0}^{\infty} \frac{t^{l}}{l !(l+1)^{k}} \\
=\sum_{m=0}^{\infty} \sum_{k=0}^{m} \sum_{j=0}^{k}\left(\begin{array}{c}
m \\
k
\end{array}\right) \frac{G_{j, \lambda}^{(F)}(u) S_{2}(k, j)}{(1-u)^{j}} B_{m-k} \frac{t^{m}}{m !} \sum_{l=0}^{\infty} \frac{t^{l}}{l !(l+1)^{k}} \\
=\sum_{n=0}^{\infty} \sum_{m=0}^{n} \sum_{k=0}^{m} \sum_{j=0}^{k}\left(\begin{array}{c}
n \\
m
\end{array}\right)\left(\begin{array}{c}
m \\
k
\end{array}\right) \frac{G_{j, \lambda}^{(F)}(u) S_{2}(k, j)}{(1-u)^{j}} \frac{B_{m-k}}{(n-m+1)^{k}} \frac{t^{n}}{n !} .
\end{gathered}
$$

This completes the proof of the theorem.

Theorem 6. For $k \in \mathbb{Z}$ and $n \geq 0$, we have

$$
G_{n, \lambda}^{(F, k)}(x+1 ; u)-u G_{n, \lambda}^{(F, k)}(x ; u)=\sum_{m=0}^{n} \sum_{l=1}^{m+1}\left(\begin{array}{c}
n \\
m
\end{array}\right) \frac{S_{1}(m+1, l)(1-u)^{m+1}}{l^{k-1}(m+1)}(x)_{n-m, \lambda} .
$$

Proof. By Definition 1 and formula (2.5), we see that

$$
\begin{gathered}
\sum_{n=0}^{\infty}\left(G_{n, \lambda}^{(F, k)}(x+1 ; u)-u G_{n, \lambda}^{(F, k)}(x ; u)\right) \frac{t^{n}}{n !}=\frac{\operatorname{Ei}_{k}(\log (1+(1-u) t))}{e_{\lambda}(t)-u} e_{\lambda}^{x}(t)\left(e_{\lambda}(t)-u\right) \\
=\operatorname{Ei}_{k}(\log (1+(1-u) t)) e_{\lambda}^{x}(t) \\
=\sum_{m=0}^{\infty} \sum_{l=1}^{m+1} \frac{S_{1}(m+1, l)(1-u)^{m+1}}{l^{k-1}(m+1)} \frac{t^{m+1}}{m !} \sum_{n=0}^{\infty}(x)_{n, \lambda} \frac{t^{n}}{n !} \\
=\sum_{n=0}^{\infty} \sum_{m=0}^{n} \sum_{l=1}^{m+1}\left(\begin{array}{c}
n \\
m
\end{array}\right) \frac{S_{1}(m+1, l)(1-u)^{m+1}}{l^{k-1}(m+1)}(x)_{n-m, \lambda} \frac{t^{n}}{n !}
\end{gathered}
$$

which gives the asserted result in (2.8).

\section{Conclusion}

In the present paper, we have considered type 2 degenerate poly-Frobenius-Genocchi polynomials and numbers by means of the polylogaritm function. Then, we have investigated diverse explicit expressions and some identities for those numbers and polynomials.

\section{References}

[1] Acikgoz, M., Simsek, Y. On multiple interpolation functions of the Nörlund-type $q$-Euler polynomials. Abstract and Applied Analysis Volume 2009, Article ID 382574, 14 pages.

[2] Kumam, W., Srivastava, H.M., Wani, S.A., Araci, S., Kumam, P. Truncated-exponential-based Frobenius-Euler polynomials. Adv. Differ. Equ. 2019, 530 (2019).

[3] Araci, S., Acikgoz, M. Construction of Fourier expansion of Apostol Frobenius-Euler polynomials and its applications. Adv. Differ. Equ. 2018, 67 (2018).

[4] Araci, S. Novel identities for $q$-Genocchi numbers and polynomials. J. Funct. Spaces Appl. 2012 (2012), Article ID 214961.

[5] Araci, S. Novel identities involving Genocchi numbers and polynomials arising from applications of umbral calculus. Appl. Math. Comput. 233 (2014), 599-607. 
[6] Araci, S., Acikgoz, M., Sen, E. On the von Staudt-Clausen's theorem associated with $q$-Genocchi numbers. Appl. Math. Comput. 247 (2014), 780-785.

[7] Cangul, I.N.; Kurt, V.; Ozden, H.; Simsek, Y. On the higher-order $w$-q-Genocchi numbers. Adv. Stud. Contemp. Math. (Kyungshang) 2009, 19, 39-57.

[8] Dolgy, D.V., Jang, L.C. Some identities on the poly-Genocchi polynomials and numbers. Symmetry 2020, $12,1007$.

[9] Duran, U., Acikgoz, M., Araci, S. Symmetric identities involving weighted $q$-Genocchi polynomials under S4. Proc. Jangjeon Math. Soc. 2015, 18, 445-465.

[10] Duran, U., Acikgoz, M., On generalized degenerate Gould-Hopper based fully degenerate Bell polynomials, Iranian Journal of Mathematical Sciences and Informatics, 2020, 21(3), 243-257.

[11] Duran, U., Acikgoz, M., On Degenerate Truncated Special Polynomials, Mathematics, 2020, 8(1), 144; https://doi.org/10.3390/math8010144.

[12] Duran, U., Acikgoz, M., Araci, Construction of the Type 2 Poly-Frobenius-Genocchi Polynomials with Their Certain Applications, preprint.

[13] Eastham, M. S. P. On Polylogarithms. Proc. Glasgow Math. Assoc. 6, 1964, 169-171.

[14] He, Y., Kim, T. General convolution identities for Apostol-Bernoulli, Euler and Genocchi polynomials. J. Nonlinear Sci. Appl. 9 (2016), 4780-4797.

[15] Jang, L.-C., Lee, J. G., Rim, S.-H., Park. S., On the degenerate Frobenius-Genocchi polynomials, Global Journal of Pure and Applied Mathematics, 2015, 11(5), 3601-3613.

[16] Khan, N., Usman, T., Nisar, K.S. A Study of Generalized Laguerre Poly-Genocchi Polynomials. Mathematics $2019,7,219$.

[17] Khan, W. A., Srivastava, D. On the generalized Apostol-type Frobenius-Genocchi polynomials. Filomat, 33:7 (2019), 1967-1977.

[18] Kim, D.-S., Kim, T. A note on polynexponential and unipoly functions, Russ. J. Math. Phys, 26(1), 2019, 40-49.

[19] Kim, T., Kim, D.S., Kwon, J.K., Kim, H.Y. A note on degenerate Genocchi and poly-Genocchi numbers and polynomials. J. Inequal. Appl. 2020, 2020, 110

[20] Kim, T., Kim, D.S., Kwon, J.K., Lee, H.S. Degenerate polyexponential functions and type 2 degenerate poly-Bernoulli numbers and polynomials. Adv. Differ. Equ. 2020, 2020, 168

[21] Kim, T., Kim, D.S. Degenerate polyexponential functions and degenerate Bell polynomials. J. Math. Anal. Appl. 487(2), $124017(2020)$.

[22] Kim, T., Rim, S. H., Kim, H.Y., Jang, L.C. Degenerate Poly-Bernoulli numbers and polynomials, Informatica, 2020, 31(3), $2-8$.

[23] Kim, T. Some identities for the Bernoulli, the Euler and the Genocchi numbers and polynomials. Adv. Stud. Contemp. Math. 2010, 20, 23-28.

[24] Kim. T.; Kim, D.S. Degenerate Bernstein polynomials. RASCAM. 2018, doi: 10.1007/s13398-018-0594-9.

[25] Kim. T.; Kim, D.S. Degenerate central Bell numbers and polynomials. RACSAM. 2019, doi: 10.1007/s13398-019-00637-0.

[26] Kim T., Jang, L.-C., Kim, D. S., Kim, H. Y., Some Identities on Type 2 Degenerate Bernoulli Polynomials of the Second Kind, Symmetry, 2020, 12(4), 510; https://doi.org/10.3390/sym12040510.

[27] Kim, T., Khan, W. A., Sharma, S. K., Ghayasuddin, M., A Note on Parametric Kinds of the Degenerate Poly-Bernoulli and Poly-Genocchi Polynomials, Symmetry, 2020, 12(4), 614; https://doi.org/10.3390/sym12040614.

[28] Kurt, B., Simsek, Y. On the Hermite based Genocchi polynomials. Adv. Stud. Contemp. Math. (Kyungshang) 2013, 23, $13-17$.

[29] Lee, D.S., Kim, H.K., Jang, L.-C. Type 2 Degenerate Poly-Euler Polynomials, Symmetry, 2020, $12,1011$.

[30] Lim, D., Some identities of degenerate Genocchi polynomials, Bull. Korean Math. Soc., 2016, 53(2), 569-579.

[31] Qin, S. Fully Degenerate Poly-Genocchi polynomials, Pure Mathematics, 2020, 10(4), 345-355.

[32] Srivastava, H.M., Kurt, B., Simsek, Y. Some families of Genocchi type polynomials and their interpolation functions. Integral Transf. Spec. Funct. 2012, 23, 919-938.

[33] Wani, S. A., Khan, S., Nahid, T. Gould-Hopper based Frobenius-Genocchi polynomials and their generalized form, Afrika Matematika, 2020, doi: 10.1007/s13370-020-00804-2.

[34] Yasar, B.Y., Ozarslan, M. A. Frobenius-Euler and Frobenius-Genocchi polynomials and their differential equations, New Trends in Mathematical Sciences, 3(2) (2015) 172-180. 